\title{
To find the Efficacy of Therapeutic Laser for Piriformis Syndrome
}

\author{
${ }^{1}$ Surendra Ojha, ${ }^{2}$ Chandan Jailiya
}

\begin{abstract}
Introduction: Excessive static and dynamic muscle work/ action of the piriformis causes Piriformis syndrome (PS). The sciatic nerve runs on bilateral side of posterior hip or thigh just closed to piriformis muscles or sometimes just passes through it. In case of PS, patients feel sharp radiating pain and paresthesia and muscle weakness in lower quadrants of limbs including low back, hip, and ankle.
\end{abstract}

\begin{abstract}
Aims and objectives: The aim of the study was to compare and observe the effect of light amplification by stimulated emission of radiation (LASER) therapy and ultrasound therapy in case of PS. The purpose of this study was to investigate the effectiveness of laser therapy for the physiotherapy rehabilitation to restore range of motion (ROM) of lower limb and decrease pain and disability in PS.
\end{abstract}

Materials and methods: Thirty patients were taken for this study. The mean age in group I and group II were 28.2 \pm 5.116 and $29.93 \pm 4.793$ respectively, and the patients were referred from orthopedic outpatient physiotherapy department of Mahatma Gandhi Hospital, Jaipur, Rajasthan, India. The duration of study was of 3 weeks. Baseline measurements of pain intensity, lower extremity functional disability, and ROM of all the subjects were measured using the visual analog scale (VAS), Lower Extremity Functional Scale, and goniometer respectively, and recorded as per test data for statistical analysis.

Results and conclusion: The results have shown that clinically there is a significant difference in the subjects treated with low-level laser and ultrasound efficient in treating pain, ROM, and function in subjects with PS. Between-the-group comparison of values of VAS readings for pain was found to be statistically significant $(0.0001)$ for ROM, but was not found statistically significant for function.

Keywords: External rotation, Functional lower extremity scale, Laser, Lower limb, Piriformis syndrome, Range of motion, Visual analog scale.

How to cite this article: Ojha S, Jailiya C. To find the Efficacy of Therapeutic Laser for Piriformis Syndrome. J Mahatma Gandhi Univ Med Sci Tech 2017;2(1):14-17.

${ }^{1}$ Vice Principal and Assistant Professor, ${ }^{2}$ Physiotherapist

1,2Mahatma Gandhi Physiotherapy College, Mahatma Gandhi University of Medical Sciences \& Technology, Jaipur, Rajasthan India

Corresponding Author: Surendra Ojha, Vice Principal and Assistant Professor, Mahatma Gandhi Physiotherapy College Mahatma Gandhi University of Medical Sciences \& Technology Jaipur, Rajasthan, India, e-mail: therapistojha@gmail.com

\section{Source of support: Nil}

Conflict of interest: None

\section{INTRODUCTION}

\section{Background}

Excessive static and dynamic muscle work/action of the piriformis causes Piriformis syndrome (PS).$^{1-3}$ The sciatic nerve runs on bilateral side of posterior hip or thigh just closed to piriformis muscles or sometimes just passes through it. In case of PS, patients feel sharp radiating pain and paresthesia and muscle weakness in lower quadrants of limbs including low back, hip, and ankle. Yeoman ${ }^{4}$ was the first to describe pain in the sciatic distribution to PS. With a few exceptions, the literature on PS includes only isolated case reports.

\section{Pathophysiology}

There are two components contributing to the clinical presentation, namely somatic and neuropathic. The somatic component underlying PS is a myofascial pain syndrome of the piriformis muscle. The symptomatology of the PS can also be contributed from a few muscles in the vicinity. They are the small external rotators of the hip (obturator internus, in particular, because it is partly an intra pelvic muscle and partly a hip muscle) and the hamstring muscles (through activation and perpetuation of trigger points). Previous gluteal trauma can cause sciatica-like pain in case of compression of sciatic nerve at their course and piriformis foramen known as components of neuropathic presentation.

\section{Etiological Factors}

Gluteal trauma in the sacroiliac or gluteal areas (possibly several years previously), myofascial trigger ${ }^{5}$ points, hypertrophy, and spasm of the piriformis muscle secondary to laminectomy, abscess, hematoma, myositis, bursitis of the piriformis muscle, neoplasms in the area of the infrapiriformis foramen, colorectal carcinoma, neuroma of the sciatic nerve, episacroiliac lipoma, intragluteal injection, femoral nailing, myositis ossificans of the piriformis muscle, Klippel-Trénaunay syndrome are the etiological factors. 


\section{Clinical Features}

Clinical features included pain with sitting, standing, or lying longer than 15 to 20 minutes, pain or paresthesia radiating from the sacrum through the gluteal area and down posterior aspect of thigh, usually stopping over the thigh, pain improves with ambulation and worsens with no movement, pain when rising from seated or squatting position, contralateral sacroiliac pain, numbness in foot, weakness in ipsilateral lower extremity. ${ }^{6,7}$ Piriformis syndrome is commonly associated with sports that require a lot of running, change of direction, or weight-bearing activity.

The pathomechanical changes contributing to PS are pathological changes in ankle foot complex, normal gait patterns and deviations, and bad posture or sitting habits.

\section{Diagnosis}

The diagnosis of PS is usually based on the findings of clinical examination, and some degree of confirmation is attributed to successful treatment targeted at the piriformis muscle. Clinical tests are aimed at assessing sciatic nerve or irritability of piriformis. ${ }^{8,9}$

\section{Straight Leg Raising}

This is primarily a test of sciatic nerve irritation, and is useful if it mimics the patient's symptoms at a relatively low angle $\left(<45^{\circ}\right) \cdot{ }^{10}$

Prone knee bending (PNB) patient in prone and passively bend one leg in knee flexion.

FABER test and others spinal cord compression test eg. slump test .

\section{Physical Therapy Protocol for Patients with PS: Stretching and Strengthening Exercises}

Stretching and strengthening exercises are done with weights or with thera-bands. Muscle isometric and isotonic exercise for hip and back muscle are adhered using proprioceptive neuromuscular facilitation techniques in different patterns. Ultrasound of 2.0 to $2.5 \mathrm{~W} / \mathrm{cm}^{2}$ is applied in broad strokes longitudinally along the piriformis muscle from the conjoint tendon to the lateral edge of the greater sciatic foramen for 10 to 14 minutes with moist heat, or sometimes cold spray at the same location for 10 minutes.

\section{MATERIALS AND METHODS}

\section{Pretest-Posttest Experimental Design of Study}

A sample of 30 subjects was randomly divided into two groups (15 each) among the subjects who were referred from Orthopedic Department to the outpatient Physiotherapy Department of Mahatma Gandhi Hospital,
Jaipur, Rajasthan, India. The subjects were selected by convenient sampling method based on initial baseline assessment and diagnosis of their condition, inclusion criteria, exclusion criteria, and history of referred pain caused by PS.

Baseline measurements of pain intensity, lower extremity functional disability, and range of motion (ROM) of all the subjects were measured using the visual analog scale (VAS), Lower Extremity Functional Scale, and Goniometer respectively, and recorded as per test data for statistical analysis. ${ }^{11}$

\section{Protocol}

Subjects were assigned to groups I and II randomly and distributed in each group comprising 15 patients. Group I was given light amplification by stimulated emission of radiation (LASER), postinhibition technique, stretching, and moist heat. Group II was given muscle energy technique (MET), moist heat, stretching, and ultrasound (3 MHz).

Data were noted at baseline, day 0 , and of 1 st week and 2 nd week and follow-up after 2 weeks. Pain, disability index, and ROM of lower extremity were reassessed.

\section{Procedure}

A written informed consent was received from 30 subjects of PS. The study will be performed by assigning 15 subjects from a group of 15 to either of the two training groups. Pretest measurements of variables will be taken with the help of VAS and using ROM by Goniometer.

Group I consists of experimental group of 15 subjects treated with low-level laser therapy (LLLT). ${ }^{12}$ Lowlevel laser therapy was applied to the skin projection at the anatomical site of the piriformis muscle with the following parameters: Wavelength $905 \mathrm{~nm}$, frequency $5000 \mathrm{~Hz}$, power density of $12 \mathrm{MW} / \mathrm{cm}^{2}$, treatment time 120 seconds, and postinhibition energy technique for 7 to 10 seconds hold.

Group II consists of control group of 15 subjects that were given ultrasound therapy $(3 \mathrm{MHz})$, postinhibition MET 7 to 10 seconds hold, and three times stretching of piriformis muscles 30 seconds hold, and stretching and moist heat. Intensity of exercises will be given four times in a week for 2 weeks.

\section{DATA ANALYSIS AND RESULTS}

The paired t-test, means, and standard deviations were used. Differences between treatment groups in change scores at the baseline and at the end of each treatment sessions over a period of 2 consecutive weeks were analyzed with the paired t-test. 


\section{Between-the-group Comparison of Pain, ROM, and Function}

0 Week: The mean value for pain, ROM, and function measured using VAS, goniometer, and FLES respectively, of participants in groups I and II measured at 0 week came out to be $7.333 \pm 0.617$ and $7.27 \pm 0.799$ for pain, $16.67 \pm 6.029$ and $14.33 \pm 4.577$ for $R O M$, and $21.67 \pm 8.381$ and $25.67 \pm 6.510$ for function respectively (Table 1 and Graph 1).

Between-the-group comparison of values of pain, $\mathrm{ROM}$, and function was done using paired t-test, with the level of significance $p$ set at 0.05 . The comparison of VAS readings for pain between groups I and II was not found to be statistically significant $(0.0001)$, which states the reliability of data at baseline.

Second Week: The mean value for pain, ROM, and function measured using VAS, goniometer, and FLES respectively, of participants in groups I and II measured at 2 nd week came out to be $2.00 \pm 0.000$ and $2.67 \pm 0.617$ for pain, $39.67 \pm 2.968$ and $34.00 \pm 3.381$ for $R O M$, and $58.00 \pm 9.964$ and $52.00 \pm 7.512$ for function respectively (Table 2 and Graph 2).

Between-the-group comparison of values of pain, $\mathrm{ROM}$, and function was done using paired t-test, with the level of significance $\mathrm{p}$ set at 0.05 . The comparison of VAS readings for pain between groups I and II was found to be statistically significant (0.0001) for pain and ROM, but was not found statistically significant for function.

Table 1: Mean value of pain, ROM, and FLES between the two groups at 0 week

\begin{tabular}{llll}
\hline & Group I & Group II & $p$-value \\
\hline Pain & $7.333 \pm 0.617$ & $7.27 \pm 0.799$ & 0.827 \\
ROM & $16.67 \pm 6.029$ & $14.33 \pm 4.577$ & 0.398 \\
FLES & $21.67 \pm 8.381$ & $25.67 \pm 6.510$ & 0.145 \\
\hline
\end{tabular}

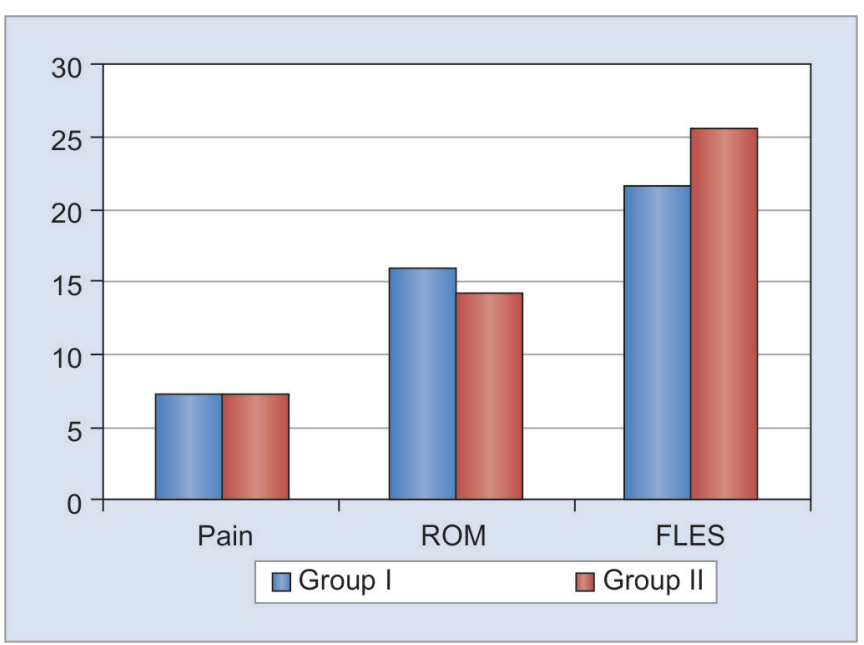

Graph 1: Mean value at 0 week

\section{DISCUSSION}

Based on the results of our study, there was a significant difference found in the efficacy of low-level laser over ultrasound. In this study, our aim was to find out the efficacy of LLLT on different parameters, namely pain, ROM, and function in PS. The study was a clinical trial performed on 30 male and female subjects diagnosed with PS with an intervention including LLLT, self-stretching technique, MET, and moist heat for group I and ultrasound therapy, self-stretching technique, MET, and moist heat for group II.

For pain in group I, a statistically significant difference was found from preintervention values to second week postintervention values. Similarly, ROM of both groups showed statistically significant difference between preintervention and second week postintervention values. In the same direction, both the groups I and II showed a statistically significant difference in preintervention and second week postintervention values.

Though the analgesic effects of laser in musculoskeletal disorder are being debated, still there are few possible suggested mechanisms of laser therapy of pain and function of lower back, one of which may be through its positive effects on chrondrocyte proliferation and matrix synthesis. Laser has also been suggested to increase the activation of cytoplasmic enzymes, oxygen consumption, adenosine triphosphate production, and the synthesis of nucleic acid and proteins. It has also been reported that laser therapy

Table 2: Mean value of pain, ROM, and FLES between the two groups at 2 nd week

\begin{tabular}{llll}
\hline & Group I & Group II & $p$-value \\
\hline Pain & $2.00 \pm 0.000$ & $2.67 \pm 0.617$ & 0.001 \\
ROM & $39.67 \pm 2.968$ & $34.00 \pm 3.381$ & 0.000 \\
FLES & $58.00 \pm 9.964$ & $52.00 \pm 7.512$ & 0.070 \\
\hline
\end{tabular}

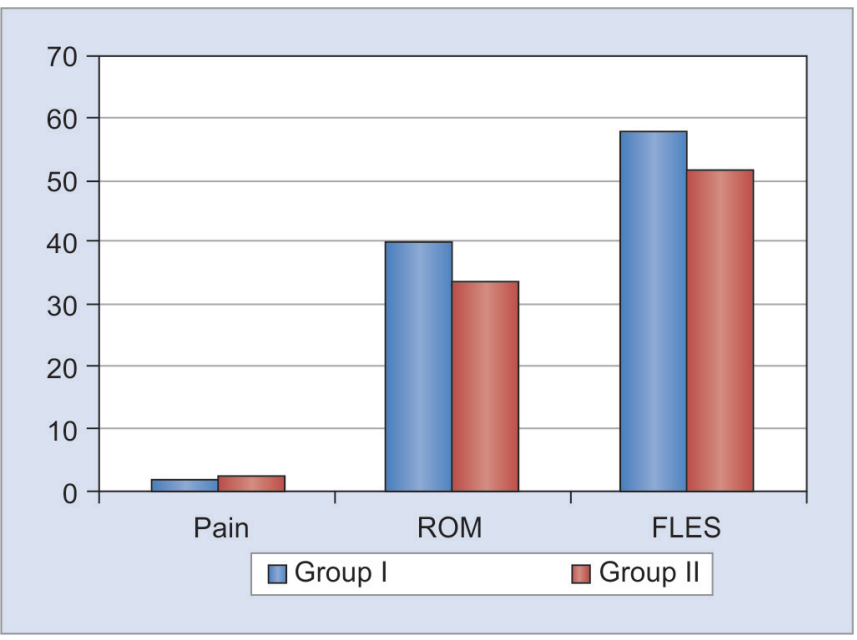

Graph 2: Mean value at 2nd week 
had antiinflammatory and antiedematous action owing to its influence, reducing prostaglandin synthesis. In a clinical trial, laser was demonstrated to have a spasmolytic effect in muscles. Thus, laser seems to break the pain-spasm-pain cycle by dual effect, therefore making the muscles more flexible, in turn increasing the ROM of adjoining joints. Another probable mechanism for pain reduction and increase in the ROM, and thus improved function, could be by increased serotonin and endogen opioid oscillation, which can be effective on serotonin metabolism.

On the comparison of pain, ROM, and function in groups I and II, a significant difference was found in the values of pain and ROM postintervention second week. Between both groups I and II, reduced mean of VAS rating for pain was found in group I and similarly increased mean for ROM was found for the participants of group I over group II.

\section{CONCLUSION}

By virtue of this study, we can conclude that LLLT is efficient in treating pain, ROM, and function in subjects with PS. Also, the results have shown that clinically there is a significant difference in the subjects treated with LLLT and ultrasound.

\section{REFERENCES}

1. Papadopoulos EC, Khan SN. Piriformis syndrome and low back pain: a new classification and review of the literature. Orthop Clin North Am 2004 Jan;35(1):65-71.
2. Parziale JR, Hudgins TH, Fishman LM. The piriformis syndrome. Am J Orthop (Belle Mead NJ) 1996 Dec;25(12): 819-823.

3. Retzlaff EW, Berry AH, Haight AS, Parente PA, Lichty HA, Turner DM, Yezbick AA, Lapcevic JS, Nowland DJ. The piriformis muscle syndrome. J Am Osteopath Assoc 1974 Jun;73(10):799-807.

4. Yeoman $\mathrm{W}$. The relation of arthritis of the sacro-iliac joint to sciatica: with one analysis of 100 Cases. Lancet. 1928;2:1119-23

5. Travell, JG.; Simons, DG. Myofascial pain and dysfunction: the trigger point manual - the lower extremities. Vol. 2. Baltimore: Lippincott Williams \& Wilkins; 1992. p. 186-214.

6. Hollinshead, WH. Buttock, hip joint and thigh. In: Hollinshead, WH., editor. Anatomy for surgeons - the back and limbs. 3rd ed. New York: Harper and Row; 1982. p. 666-668, 702.

7. McCrory P, Bell S. Nerve entrapment syndromes as a cause of pain in the hip, groin and buttock. Sports Med 1999 Apr;27(4):261-274.

8. Durrani Z, Winnie AP. Piriformis muscle syndrome: an underdiagnosed cause of sciatica. J Pain Symptom Manage 1991 Aug;6(6):374-379.

9. Edwards PH Jr, Wright ML, Hartman JF. A practical approach for the differential diagnosis of chronic leg pain in the athlete. Am J Sports Med 2005 Aug;33(8):1241-1249.

10. Silver JK, Leadbetter WB. Piriformis syndrome: assessment of current practice and literature review. Orthopedics 1998 Oct;21(10):1133-1135.

11. Touliopolous S, Hershman EB. Lower leg pain. Diagnosis and treatment of compartment syndromes and other pain syndromes of the leg. Sports Med 1999 Mar;27(3):193-204.

12. Ferreira DM, Zângaro RA, Villaverde AB, Cury $Y$, Frigo L, Picolo G, Longo I, Barbosa DG. Analgesic effect of He-Ne $(632.8 \mathrm{~nm})$ low-level laser therapy on acute inflammatory pain. Photomed Laser Surg 2005 Apr;23(2):177-181. 